\title{
Topical issue on Novel Quantum Phases and Mesoscopic Physics in Quantum Gases
}

Since the achievement of Bose-Einstein condensation (BEC) and superfluidity with atomic Fermi gases, ultracold quantum gases have become a very versatile testing ground for quantum many-body theories. The amazing experimental possibilities which allow one to change the dimensionality of the system, introduce disorder in a controlled way, and create a whole variety of strongly correlated states, have made the connection between the physics of ultracold quantum gases and condensed matter physics stronger and stronger. This connection has been especially taken into consideration in the selection of the papers of this topical issue of the European Physical Journal B. In addition, attention has been also devoted to the recent activities aimed to the realization of BEC in other condensed matter systems (e.g. polaritons, magnons in Helium-3), which create further links to the condensed matter community.

A topic of major relevance at the moment concerns the presence of disorder, both for light and matter-waves. The very recent realization of Anderson localisation of non interacting matter-waves with Bose-Einstein condensates in quasi-one-dimensional geometries, opens up the very promising possibility of understanding the problem of disorder in the presence of interactions and/or in higher dimensionality. Using optical lattices it is also possible to create several geometries suited to the study of low dimensional systems, quantum phase transitions, and the creation of supersolid and paired phases of matter. Especially fascinating results arise from the possibility of tuning the atomic interactions. Using of Fano-Feshbach resonances to change the magnitude and sign of the s-wave scattering length, has allowed, e.g., the observation of collapsing Bose condensates, of the crossover from a BEC to a Bardeen-Cooper-Schrieffer-type transition and the controlled creation of molecules. More recent experiments have demonstrated that the range of the interactions can also be manipulated. Dipole interactions with long-range anisotropic character have been observed in ${ }^{52} \mathrm{Cr}$ atoms and this has opened up a rich and active research field. The reduced dimensionality has also become a very useful tool for making possible the realization of the Berezinskii-Kosterlitz-Thouless superfluid phase transition occuring in two-dimensional systems. The target list of the present topical issue includes various contributions on the subjects above but also contributions on the quantum magnetic phases of atoms in optical lattices (ordered or spin liquids), the problem of spin squeezing in a bimodal condensate, and the static properties of Bose-Fermi mixtures. Many of these systems pose longstanding theoretical and experimental condensed matter questions, which might find in ultracold atomic systems a first non controversial answer.

This topical issue contains original results on these various most relevant research directions in the field of ultracold gases. The papers originate from a selection of works presented at the Fourth International Workshop "Theory of Quantum Gases and Quantum Coherence" and at the CNRS École Thématique "Physique Mésoscopique avec les gaz quantiques" held in Grenoble (France) from June the $3^{\text {rd }}$ to June the $7^{\text {th }}$, 2008. All papers have been peer reviewed following the usual standards and procedures of The European Journal of Physics B. We would like to thank the Chief Editors for giving us the possibility of publishing this topical issue and the support of the Editorial Staff in the persons of Nicolas Puyaubreau and Véronique Condé, of the Editorial Board in the person of Kurt Schönhammer, and the Production Staff in the person of Ariana Fuga.

This Workshop was made possible thanks to the financial support of the European Science Foundation - within the Programme "Quantum Degenerate Dilute Systems", the Centre National de la Recherche Scientifique (CNRS), the Laboratoire de Physique et Modélisation des Milieux (Grenoble), the "École Doctorale de Physique" (Grenoble), The "Centre de Théorie en Physique de Grenoble", the "BEC-CNR-INFM Research and Development Center on BoseEinstein Condensation" in Trento, the Department of Physics "E.R. Caianiello" (Salerno) and the "groupement de recherche" IMCODE. 